Recibido: mayo de 2017

Aprobado: julio de 2017

\section{LA CIUDAD IDEAL DE ROBERT OWEN. UN ESCENARIO PLANIFICADO DEL SOCIALISMO UTÓPICO*}

\author{
Carlos Humberto Gómez Arciniegas** - Universidad Santo Tomás, Colombia
}

DOI: https://doi.org/10.15332/rev.m.v14i0.2173

Vista de New Lanark (Escocia), donde Robert Owen llevó a cabo su primer experimento social de "pueblos cooperativos". Actualmente declarado patrimonio de la Humanidad por la UNESCO.

Fuente: https://www.visitheritage.co.uk

* Artículo de reflexión.

* Arquitecto por la Universidad Santo Tomás Bucaramanga, Colombia; especialista en Planificación del Territorio para el Desarrollo por el Politécnico di Milano, Italia - Université de Paris I, Pantheon-Sorbonne, Paris, Francia $\mathrm{MSc}$ en Relaciones Internacionales y Estudios Estratégicos con énfasis en Políticas de Desarrollo por Lancaster Uinversity, Inglaterra, y $\mathrm{PhD}$ en Planificación Urbana, Territorial y Ambiental por el Politécnico di Milano, Italia. Docente investigador, líder de la línea de Investigación en Planificación y Gestión del Territorio adscrita al grupo de Investigaciones de la Facultad de Arquitectura de la Universidad Santo Tomás Bucaramanga. Correo electrónico: carlos.gomez@ustabuca.edu.co

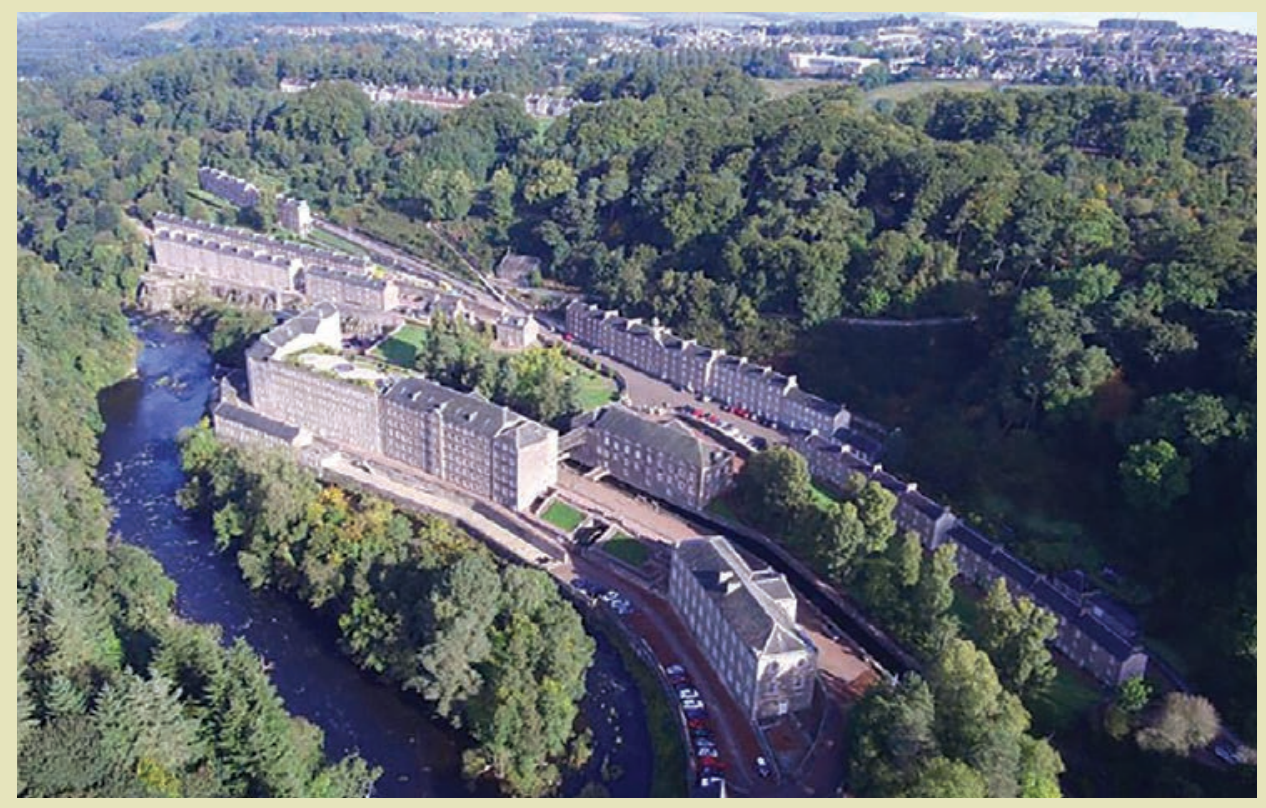

\section{RESUMEN}

El éxito económico de algunos emprendedores británicos durante los años en que Inglaterra sufrió una grave crisis económica y el desempleo alcanzó niveles alarmantes, los condujo a pensar en transformaciones de índole social. Pionero entre estos fue el galés Robert Owen quien concibió una comunidad avanzada que residiría en una ciudad ideal a partir de su éxito como empresario en Escocia. Propuso un modelo de convivencia ideal en un edificio en un entorno rural donde un grupo de personas trabajaría y viviría felizmente. Sus postulados se basaban en la necesidad de encontrar una ocupación rentable para pobres y desempleados, apoyados en los cambios y adelantos característicos de la revolución industrial. El presente artículo se acerca inicialmente a la fundamentación de Owen para promover cambios profundos en la sociedad de esa época. Seguidamente, pasa a describir cómo se plasmaron sus ideas en un proyecto que para esos tiempos fue considerado como utópico, diseñado por un arquitecto, de quien también se hace referencia, en donde se buscaba el confort, el desarrollo humano y el mejoramiento de la producción en condiciones favorables y equilibradas. Por tanto, el artículo escudriña en la propuesta de Owen, deteniéndose en especificaciones formales y funcionales del proyecto. La parte final se refiere al ocaso de la utopía de este pre urbanista $y$ al legado que de una u otra forma dejaron sus ideales a la sociedad actual.

\section{PALABRAS CLAVE}

Ciudad ideal, modelo progresista, Owen, socialismo utópico, sociedad autosostenible, planificación. 


\section{THE IDEAL CITY OF ROBERT OWEN. A PLANNED SCENARIO OF UTOPIAN SOCIALISM}

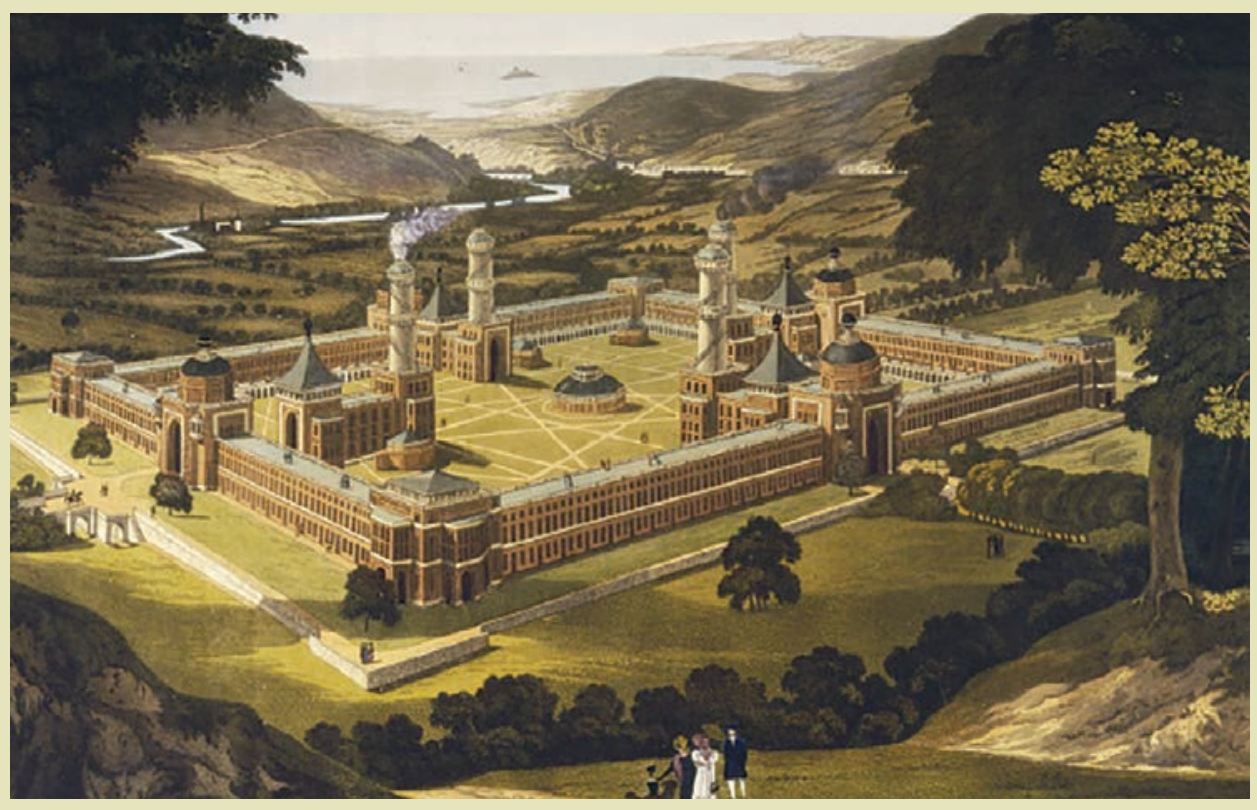

Representación gráfica del proyecto de New Harmony de Robert Owen para su modelo de ciudad ideal, el cual debería iniciarse en Indiana, Estados Unidos. Fuente: $h t t p: / / w w w . b b c . c o m /$

\begin{abstract}
The economic success of some British entrepreneurs during the years in which England suffers a serious economic crisis and unemployment reaches alarming levels, led them to think about social transformations. Pioneer among these was the Welshman Robert Owen who conceives an advanced community that would reside in an ideal city from his success as an entrepreneur in Scotland. It proposed a model of ideal coexistence in a building in a rural environment where a group of people would work and live happily. Its postulates were based on the need to find a profitable occupation for the poor and the unemployed, supported by the changes and advances characteristic of the industrial revolution. This article is initially about Owen's foundation to promote profound changes in the society of that time. Then he goes on to describe how his ideas are embodied in a project that was considered utopian for those times, designed by an architect, who is also referred to, where comfort, human development and improvement of production were sought in favorable and balanced conditions. Therefore, the article scrutinizes Owen's proposal, stopping at formal and functional specifications of the project. The final part refers to the decline of the utopia of these preurbanistas and the legacy that one way or another left their ideals to the current society.
\end{abstract}

\section{KEYWORDS}

Ideal city, progressive model, Owen, utopian socialism, self-sustaining society, planning. 


\section{INTRODUCCIÓN}

El urbanismo y la urbanización masiva del siglo XIX son hechos relativamente recientes en la historia. Sin duda, la disciplina y el fenómeno están estrechamente relacionados con un lugar industrial $y$ al mismo tiempo rechazados, asumidos o reinterpretados por diferentes estudiosos y pensadores que buscaban constituir una ciudad ideal que, por lo general, nunca llegó a realizarse, o al menos, no en su totalidad. Es desde esta posición ideológica difícil de sostener, que el siglo XIX sentó las bases ideológicas de las ciudades actuales, ya sean planificadas o no. A pesar de esto, es menos apresurado afirmar que este período se conecta con los decisivos ámbitos de la filosofía y de las remembranzas, las cuales han tenido su proyección en el tiempo y han llegado a la actualidad.

Prueba de esto son las acciones realizadas por preurbanistas como Robert Owen, Charles Fourier o Jean Baptiste Godin que pueden ser retomadas, analizadas y contextualizadas en el siglo XXI, específicamente en términos de división de usos del suelo, higiene de los conjuntos habitacionales, geometría de los asentamientos, interacción entre habitantes $y$, sobre todo, la intención de equidad social. Se trata de los agitados procesos preindustriales donde la ciudad se opone al medio rural aunque sin dejar de añorar un fuerte vínculo con el mismo, relación que será retomada en el siglo XIX por una serie de activistas del urbanismo, catalogados por Choay ( 1983 ) según sus posiciones al respecto como progresistas y culturalistas, en categorías que los agrupan entre preurbanistas y urbanistas, entre estos últimos el mismo ingeniero español Ildefonso Cerdá, conocido por su plan para la ciudad de Barcelona (España) o el periodista Ebenezer Howard, admirado por sus postulados teóricos sobre las ciudades jardín.

La lista sería muy extensa por lo que el presente artículo se remite a los albores del urbanismo en el marco de la Revolución Industrial, período en que aparecen los "renovados constructores de ciudades" del siglo XIX, muchos con un sesgo completamente innovador y otros con un corte más conservacionista que se evidencia en muchos elementos de sus propuestas y que acuden al modelo de la ciudad medieval. Por tal motivo, se posa la mirada específicamente sobre el modelo progresista y se concentra en las innovadoras ideas de Robert Owen, empresario preocupado por los fenómenos que provoca la Revolución Industrial, inicialmente en Inglaterra y después en varios países europeos y que empezaron a hacerse sentir a partir del éxodo del campo a las ciudades con sus inevitables consecuencias: precarias condiciones de vida, insalubridad, diversas formas de hacinamiento y una marcada explotación del proletariado bajo los modelos de producción impuestos por la burguesía. Estas condiciones enriquecieron a muchos empresarios que solo se preocuparon por fortalecer su capital, pero es también claro que otros hombres de férrea posición económica fueron movidos por esta situación a visionarias formas de organización social.

En la fase sucesiva, se describen como se plasman sus ideas en un proyecto que para esos tiempos fue considerado como utópico, donde se buscaba el confort, el desarrollo humano y el mejoramiento de la producción en condiciones favorables y equilibradas. Por tanto, el artículo estudia la propuesta de Owen, deteniéndose en especificaciones formales y funcionales del proyecto y es por esto que se conceden los créditos pertinentes al arquitecto de Owen. La parte final resume los principales aspectos del ocaso de la utopía de este pre urbanista y el legado que de una u otra forma aún sigue vigente en nuestros días.

\section{EL SESGO PROGRESISTA}

Explorar dentro de los albores del urbanismo moderno implica volver la mirada hacia los llamados socialistas, quienes en su romántica y libre interpretación de la sociedad van a 
formular una serie de postulados e inclusive propuestas urbanísticas y arquitectónicas que aún son objeto de atención de estudios de la ciudad. Robert Owen (I77|-|858) podría considerarse como el pionero de esa corriente nacida en la primera mitad del siglo XIX y que varios estudiosos bautizarían después con el nombre de "socialismo utópico". De hecho, Federico Engels (I820 - 1895) referencia el término en los textos producidos entre 1876 y 1878 y que darían origen a subsiguientes ediciones de la obra: Del socialismo utópico al socialismo científico. Sin embargo, es Engels quien acude a su vez a un predecesor llamado Tomás Moro (1438 - 1535) quien acuñó el término "utopía" con el que bautizó una isla imaginaria dotada de una sociedad ideal de la que describirá su sistema político en su obra más famosa Utopía, publicada en I5I6. En cualquier caso, es Owen quien ante la Revolución Industrial esgrime nuevos ideales y propuestas que sentarán las bases de ulteriores proyectos como el del "Familisterio", autoría del economista francés Charles Fourier (1772-1837) que será emulado a su vez por Jean Baptiste André Godin (1817-1888) con su “Familisterio". A una escala más amplia, aparecen los postulados de ciudades más complejas como las descritas por el fisiatra inglés Benjamín Ward Richardson (1828-1896) en su obra Hygeya, una ciudad de la salud o por Étienne Cabet (1788-1856) en su obra Viaje a Icaria, pensada por el autor para ser erigida en una tierra de promisión que en ese entonces era el continente americano. Al igual que Owen, Fourier y Cabet propenderán por la abolición de propiedad privada y el uso del dinero. Aunque la mayor parte de los experimentos propuestos por estos preurbanistas sucumbieron, sobre todo en términos de una "revolución social", fundamentada en premisas ligadas al asociacionismo y el sindicalismo, el primer nombre que sobresale en la historia es el de Robert Owen quien será notablemente reconocido en los anales del movimiento obrero británico (figura I).

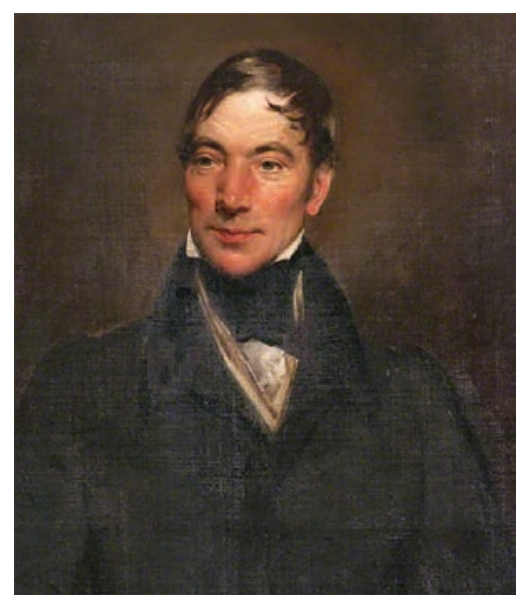

Difícil condensar su prolífica vida y sus ideales por establecer un modelo de sociedad ideal, la cual se radicaría en un proyecto no menos utópico para esa época y que buscaría exportar a los Estados Unidos. Su ímpetu se debe a muchos factores, entre estos su extracción familiar, su estudio de los filósofos franceses de la llustración como Voltaire, Diderot, Rousseau y el éxito como emprendedor en Mánchester. Antecesor de Marx (1818-1883) y Engels ( I829-1895), empresario, reformista social y político, cimenta aún más sus ideales de cambio a partir de los resultados positivos del experimento social que llevó a cabo en New Lanark (Escocia), originado por causa de las precarias condiciones de vida de la población campesina de Lanarkshire que era sometida a largas horas de trabajo y a la rutina desmoralizadora de las fábricas. A esto se le suma que los trabajadores provenían de los niveles más bajos de la sociedad por lo que el robo, la embriaguez y otros vicios eran comunes. Además, la higiene y la educación estaban notablemente descuidadas, y las familias vivían en condiciones de hacinamiento.
Figura I. Robert Owen (I77/-1858) Fuente: New Lanark Trust. 
Ante este panorama, típico de la Revolución Industrial, Owen induce a sus socios a comprar New Lanark y después de su matrimonio se muda allí. Animado por su gran éxito, conseguido entre 1800 y 1825 durante la administración de fábricas de algodón en Mánchester, primera ciudad industrial del Reino Unido, el emprendedor intentó dirigir la fábrica de New Lanark (Escocia) de acuerdo con sus principios ideales, eclipsando aquellos comerciales. En otras palabras, se preocupó por sus trabajadores y les concedió una serie de beneficios impensables para la época. Se preocupó por sus trabajadores, como residir cerca de su trabajo, pensar no solo en cómo trabaja, sino en cómo vivían, prohibió el trabajo infantil, estableció licencias por enfermedad e inclusive creó el primer preescolar en Reino Unido. Naturalmente, estableció en New Lanark una serie de reglas y pautas para sus trabajadores que amalgamó oportunamente con sus iniciativas en pro de la clase trabajadora, las cuales le garantizaron un éxito empresarial que lo llevó a formular un experimento comunitario en Estados Unidos para lo cual invita a participar a personas de diferente extracción social, entre ellos integrantes de la elite social del momento con nobles ideales, pero también aventureros y pensadores cuyo objetivo principal no era precisamente el trabajo.

Dejado a un lado los postulados sociales y filosóficos de Owen, este artículo se enfoca más en el alcance de un proyecto que fue concebido por Owen en términos arquitectónicos e inclusive como modelo experimental de planificación del territorio. El proyecto se llamaría la "Sociedad de la Nueva Armonía" y se trataría de un modelo donde no habría soberanía individual y propiedad privada. Es decir, un hecho tangible donde probar la viabilidad de sus ideas a través de una comunidad autosuficiente a partir de un plan de trabajo que buscaba la autosuficiencia comunitaria. Owen y su hijo William navegaron a Estados Unidos de América en octubre de 1824 para establecer una comunidad experimental en el Estado de Indiana. En 1825, Owen utilizó una parte de sus propios fondos para finiquitar la compra de una ciudad existente que incluía I 80 edificios y una notable extensión de tierra a lo largo del río Wabash en Indiana. El pueblo original pertenecía a un grupo religioso que lo había bautizado como Harmony (o Harmonie). Owen lo renombró como Nueva Armonía y se estableció en el pueblo para desarrollar su modelo preliminar para una comunidad utópica.

\section{UNA PROPUESTA DE PLANIFICACIÓN DEL TERRITORIO}

A partir del ideal de un nuevo orden social, constituido en su totalidad por voluntarios que crearían a su vez un nuevo mundo moral, Owen concibe en el Reino Unido un modelo de ciudad ideal, de contenidas dimensiones, pero replicable en un territorio dado, en la que todos sus habitantes trabajarían para todos y no existiría la propiedad privada.

De acuerdo con Benévolo (1994), los beneficios ofrecidos por un plan de estas características no podían ser ofrecidos a masas demasiado numerosas, sino por el contrario a grupos que oscilen entre 500 y $I 500$ personas, dando como número ideal de promedio I 200 habitantes. Además, deberían vivir en un entorno social superior, intelectual y físico que no podía lograrse en un asentamiento dado, sino que merecía un plan bien pensado y adecuadamente proyectado por lo que encarga el plan a un arquitecto de quien se hablará más adelante. Son estas las principales premisas que orientaban el plan de Owen.

La llegada al nuevo mundo de Owen y algo más de 800 personas, algunas de gran prestigio, fue vista como el nacimiento de la comunidad. En 1825 se firmó el acuerdo de compra del terreno y posteriormente en 1826 , como se expuso precedentemente, la ciudad adoptó una nueva constitución: "La Comunidad New Harmony de la lgualdad" en donde se defendía la posibilidad de desarrollar un sistema económico alternativo basado en la cooperativa. 
El proyecto "piloto" debería implantarse en el centro de una gran área de 200 acres, algo más de 800 hectáreas, gestionada por una comunidad que viviría en un terreno ligeramente elevado en un entorno netamente rural y a una distancia de 3 millas $(4,8 \mathrm{~km}) \mathrm{de}$ la ribera oriental del río Wabash. El poblado más cercano sería Mount Vernon, a 12 millas de distancia y servido por tributarios del río Ohio, también navegable como el Wabash, lo que permitía establecer oportunos canales de comunicación, por el sur con Nueva Orleans, sobre el Golfo de México y con Pittsburgh, prominente ciudad industrial de los Estados orientales sobre el Atlántico (figura 2).
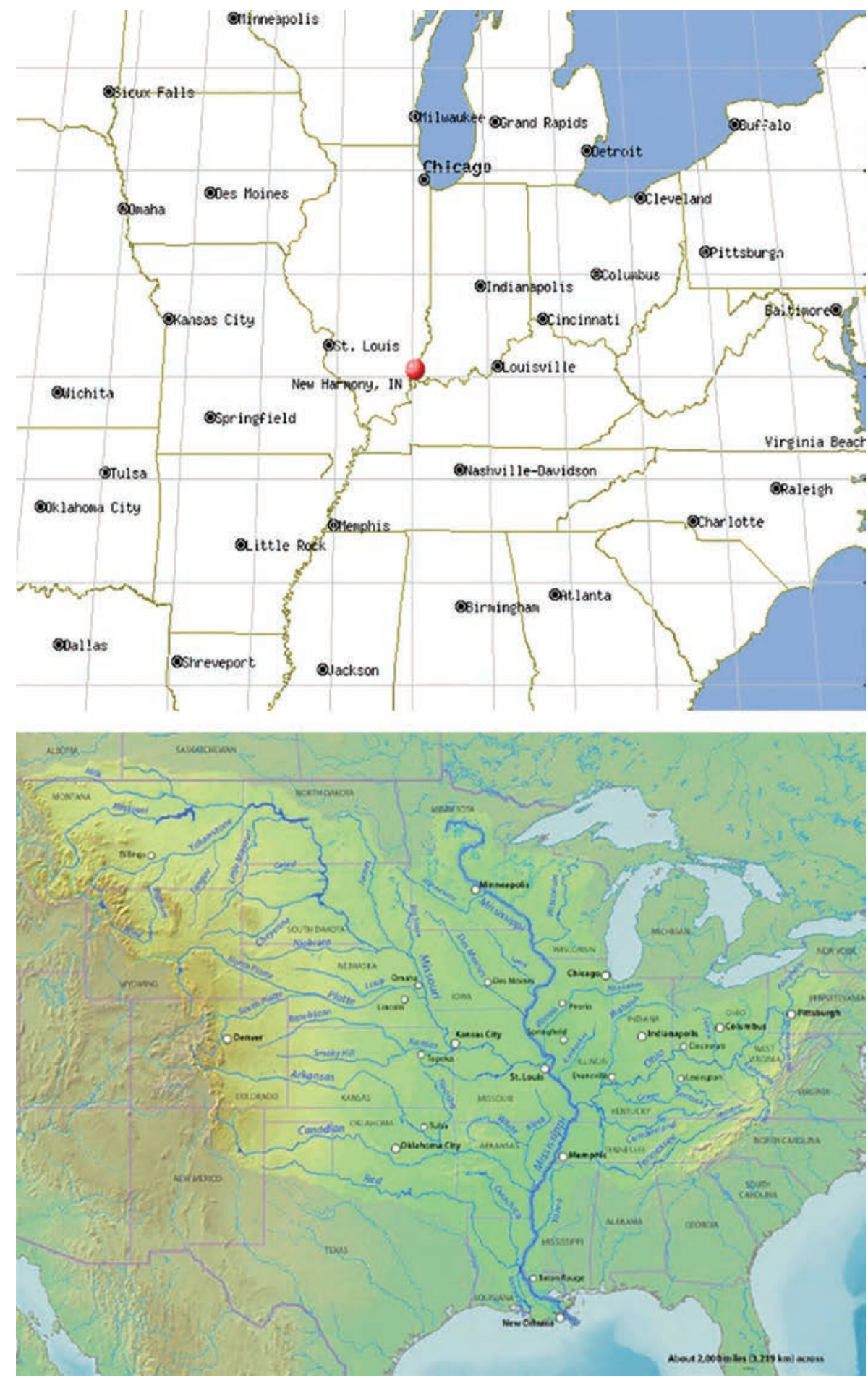

Figura 2. Localización de New Harmony Fuente: Elaboración del autor con base en cartografia de http://www.statetravelmaps.com/ Indiana/ y https://www.mississippiriverinfo.com/ map-of-the-mississippi-river/ 
Figura 3. Vista general del proyecto, según descripción hecha por Owen (1817) en el informe "Committee on the Poor Law". Se aprecian los poblados de la "unidad y la cooperación mutua" distribuidos en el territorio agrícola

Fuente: http://urbanplanning.library.cornell.edu
Es este el gran escenario natural donde se implantaría uno de los primeros "ordenamientos" equidistante a futuras "aldeas" de la misma naturaleza", según palabras de Benévolo ( 1994), que se replicarían paulatinamente. Así bien, cada aldea comprendería un gran cuadrado con capacidad para alojar I 200 personas y circundado por un terreno hasta de 1500 acres, equivalentes a 607 hectáreas aproximadamente (figura 3).

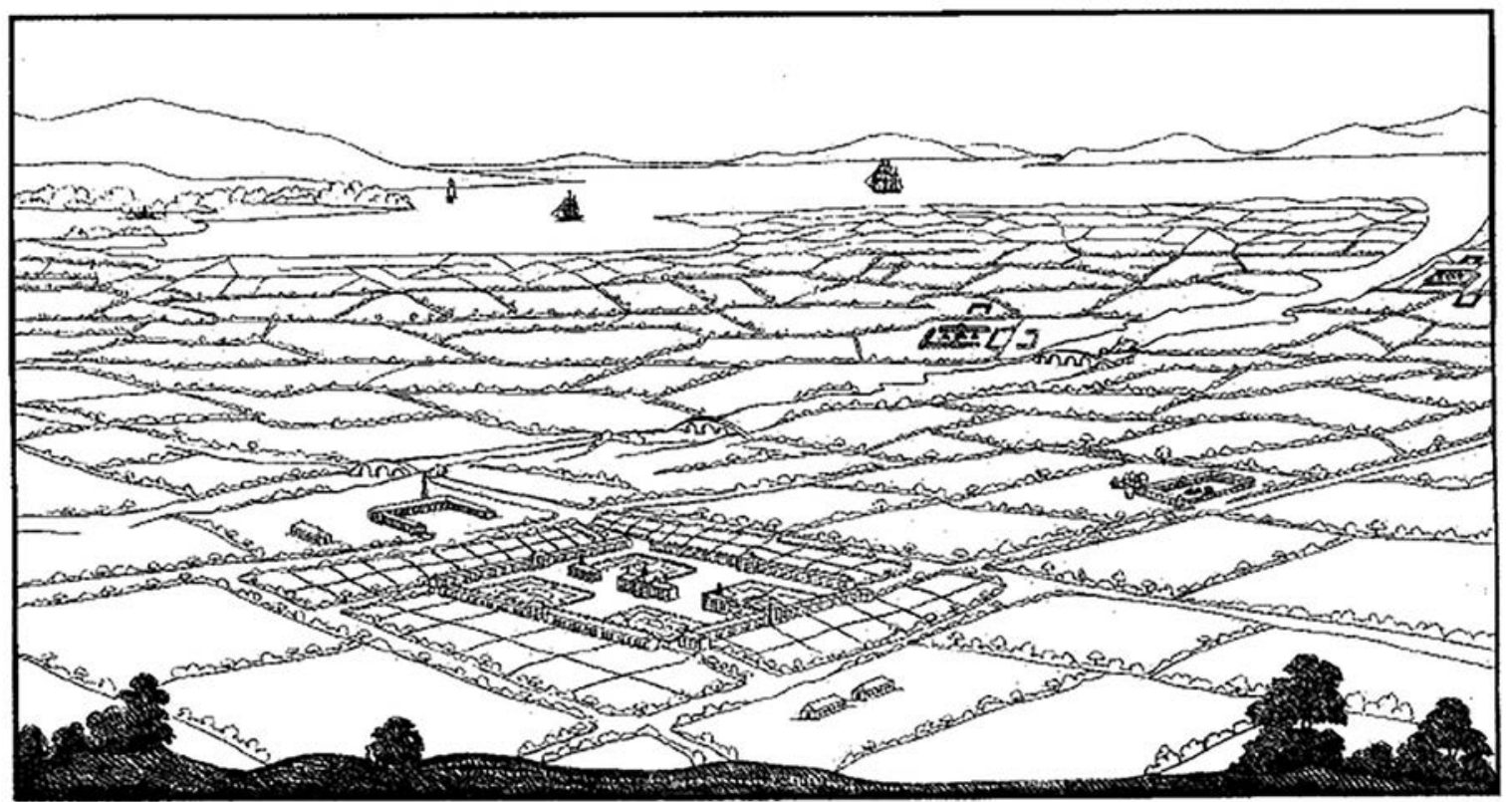

Según Reps (2002), el conjunto tendría lados de 1000 pies (304.8 m), con edificios dispuestos a lo largo del perímetro del lote para así conformar una plaza central (figura 4). En el centro y en los ángulos de dichos edificios se destacan algunos volúmenes que demarcan accesos y ciertas actividades comunitarias, los cuales a su vez articulan las alas dedicadas a las viviendas de los miembros de la comunidad. En la parte interna de la gran plaza se ubicarían sendos edificios de uso público, los jardines, huertos botánicos y otras áreas libres para actividades físicas. La totalidad del conjunto estaría en un nivel superior de la superficie natural, sobre una plataforma circundada por una explanada. El suelo del área interior estaría atravesado por diagonales que coinciden, para efectos de orientación, con un meridiano.

En líneas generales, la disposición de cada elemento obedecía atentamente a los resultados más relevantes provenientes de descubrimientos científicos de la época, todos ellos dirigidos a proporcionar confort y a contribuir al orden físico, moral e intelectual de cada individuo, jamás vistos en épocas pasadas de su vida o en determinado país. El listado de espacios y actividades propias del proyecto será analizado más delante en detalle, a partir de los mismos textos emitidos por el arquitecto de Owen: Thomas Stedman Whitwell a quien se le debe los merecidos reconocimientos por su ingenio y capacidad de plasmar en un objeto arquitectónico los ideales del filántropo que contrató sus servicios como arquitecto.

\section{El arquitecto de Owen}

Reps (2002) a través de sus estudios ofrece una breve narración de la vida profesional de Thomas Stedman Whitwell (I784- 1840), nacido en Coventry, Inglaterra. En I 806 viaja a Londres para exponer su obra en la Royal Academy of Arts para proseguir en la capital 
inglesa trabajando en una oficina de arquitectos de London Docks. Después de aumentar su experiencia profesional Whitwell regresó a West Midland, y pasó más de diez años diseñando edificios emblemáticos en Birmingham, Coventry y Warwickshire.

Según Reps, la práctica del arquitecto Whitwell no fue de hecho muy próspera, y su reputación recibió un golpe cuando uno de sus edificios, el Brunswick Theatre en el distrito londinense de Whitechapel se derrumbó apenas unos días después de que se completara. En 1819, Whitwell diseñó un proyecto a gran escala cerca de Leamington Spa que nunca se llevó a cabo.

Mejores días llegaron a su vida a partir del momento en que se conoce con Robert Owen aunque las fuentes disponibles no documentan si se ofreció como voluntario para preparar el plan que ilustraba el concepto de pueblo comunitario de Robert Owen o si este lo reclutó para este propósito. Cualquiera de las dos hipótesis son válidas dado que para ese entonces Owen contaba con una bien ganada reputación por su éxito en New Lanark y varias personas ya conocían su proyecto de aventurarse en América, por lo que este arquitecto bien pudo ser uno de sus más fervientes seguidores. En cualquier caso, no está claro cuándo Whitwell realmente coincidió con Owen, pero no parece que Whitwell haya visitado New Lanark hasta octubre de 1824, poco después de la partida de Owen a los Estados Unidos.

Donnachie (2007) y otros autores sí coinciden en que los dibujos de Whitwell proporcionaron la plantilla para el famoso modelo encargado por Owen para 1825, o sea una maqueta de seis pies $(1.83 \mathrm{~m})$ del prototipo de ciudad ideal, modelo que corresponde a la descripción que se relaciona más adelante. Registros históricos señalan que Owen logró obtener el permiso del presidente John Quincy Adams para exhibir el modelo en la Casa Blanca.

Una vez llegado a Indiana, Whitwell inspeccionó los terrenos y decidió vivir por un corto tiempo en New Harmony. Se cuenta que no mucho después de su llegada comenzó a criticar a algunos de los líderes de la comunidad y difundir chismes sobre ellos. Según Coates (200I), su única contribución, si puede llamarse así, fue un sistema de coordenadas geográficas en el que la latitud y la longitud se tradujeron en letras del alfabeto, dando nuevos nombres a los lugares. Por lo tanto, la comunidad de New Harmony a la que se unió Whitwell recibió el nombre de Feiba Peveli. Por ejemplo, en este sistema, Nueva York (40 grados, 43 minutos N y 73 grados 59 minutos W) se convirtió en Otke Notive. En cualquier caso, este invento de Whitwell según los estudiosos, no llegó a trascender, ya que solo fue visto por muchos como fruto de la gran cantidad de tiempo libre que el arquitecto tenía y dedicaba a invenciones.

Reps (2002) hace referencia a una ilustración gráfica hecha por Withwell, es decir una perspectiva, y un folleto que contenía especificaciones funcionales del proyecto, exhibidas en 1825 . Cuando se publicó el documento descriptivo del proyecto, en 1830, el experimento de New Harmony ya se había desintegrado, pues en gran medida los residentes ya se habían marcharon o se dividieron en facciones. Sin embargo, todo parece indicar que Owen llegó a considerar hacer un esfuerzo final para poner en práctica su plan en Inglaterra, o tal vez, según Reps (2002) colige, Whitwell permaneció como un fiel creyente del concepto y publicó el panfleto por su cuenta.

Lo cierto es que poco parece saberse sobre los años restantes de Thomas Whitwell después de su regreso a Inglaterra. A pesar de que continuó publicando sus ideas, incluidas algunas propuestas para calentar y ventilar viviendas, aunque no hay evidencia de que haya regresado a su trabajo de arquitectura. Los edificios por él proyectados ya cumplieron su ciclo y no queda ninguno en pie. Acorde a la información de Eighteenth Century Birmingham (20I2), solo subsisten algunos archivos que permiten hacerse una idea de una obra, escasamente documentada y levemente ligada a la historia de dicha ciudad. El arquitecto muere en 1840 y aunque Robert

M 71 REVISTA M VOL. 14 ENERO - DICIEMBRE 2017 • FACULTAD DE ARQUITECTURA • UNIVERSIDAD SANTO TOMÁS COLOMBIA 
Owen sigue siendo una figura notablemente controvertida, inspirando y dividiendo muchos historiadores y urbanistas en igual medida, el nombre de su arquitecto ha desaparecido en gran parte de la historia, junto con toda su construcción.

Owen seguiría abogando por su plan de aldea en I84I en una publicación que propone el desarrollo de "Colonias Domésticas Autosuficientes" en Inglaterra. Una vez más, preconizaría este ideal como:

Una inversión más segura y rentable para el capital, y un medio eficaz para eliminar permanentemente las causas de la ignorancia, la pobreza y la delincuencia; y desde un punto de vista materialista beneficiar a todas las clases sociales a través de una correcta aplicación, para los ahora mal dirigidos poderes de las facultades humanas y de la ciencias físicas y morales.

\section{El proyecto de New Harmony: La ciudad ideal de Owen}

Reps (2002) y otros autores, como Benévolo (1994), han rastreado en varios documentos, entre estos el reporte presentado por Owen (I8I7) al Comité de la asociación de ayuda para los pobres trabajadores e industriales, en la ciudad de Londres, las descripciones necesarias para el entendimiento de la propuesta del paralelepípedo que albergaría entre 800 a I 200 personas. El diseño arquitectónico se fundamenta en la articulación de edificios proyectados como paralelogramos, que posicionados perimetralmente en el área del lote conforman una gran plaza cuadrada. El área de terreno ocupada por los edificios, paseos y jardines del establecimiento sería de aproximadamente treinta y tres acres (I33.546 $\mathrm{m} 2$ ); de los cuales veintidós acres $(89.030,8 \mathrm{~m} 2)$ son de área libre delimitada, casi tres veces más grande que Russell Square. Allí se proponen unas líneas diagonales que deberían coincidir con un meridiano $y$, si es posible, que se extienda con algunos puntos u objetos notables del paisaje circundante. Esto garantizaría una distribución equitativa de la luz y la oscuridad, el sol y la sombra para los ocupantes de cada parte del edificio; sin olvidar la conveniencia de esta orientación para referencias astronómicas y geográficas. Era deseable, en la mayoría de los casos que el área de terreno seleccionada para la construcción de cada edificio estuviese cerca de un arroyo, lo suficientemente amplio como para suplir las necesidades domésticas del establecimiento, para asegurar su drenaje y suministrar energía suficiente para los molinos y otras instalaciones necesarias o beneficiosas para la residencia y prosperidad de los habitantes.

\section{Disposiciones generales del paralelepípedo}

De acuerdo con lo indicado anteriormente, el edificio se desarrollará en un cuadrilátero de 304.8 metros por cada lado con volúmenes que alojarín viviendas, edificios para equipamientos ubicados en las áreas centrales y en las esquinas. En cuanto al programa de las demás necesidades espaciales del proyecto, este contemplaba baterías de baños, refectorios, cocinas, fábricas de cerveza, almacenes (tiendas), oficinas, jardines botánicos y gimnasios, que gravitarían alrededor de un pórtico o logia como elemento articulador. Los espacios más importantes se interpretan según la descripción original por lo que se describen a continuación y se relacionan en la figura 4.

\section{Temperatura interna, ventilación, luz, suministro de agua, drenaje, sótano}

El área interna y en especial la dedicada a las viviendas, serían calentadas y ventiladas según principios innovadores, manteniéndose una temperatura agradable en las estaciones más frías o en las de clima más variable, por medio de calentadores de aire. El conjunto 


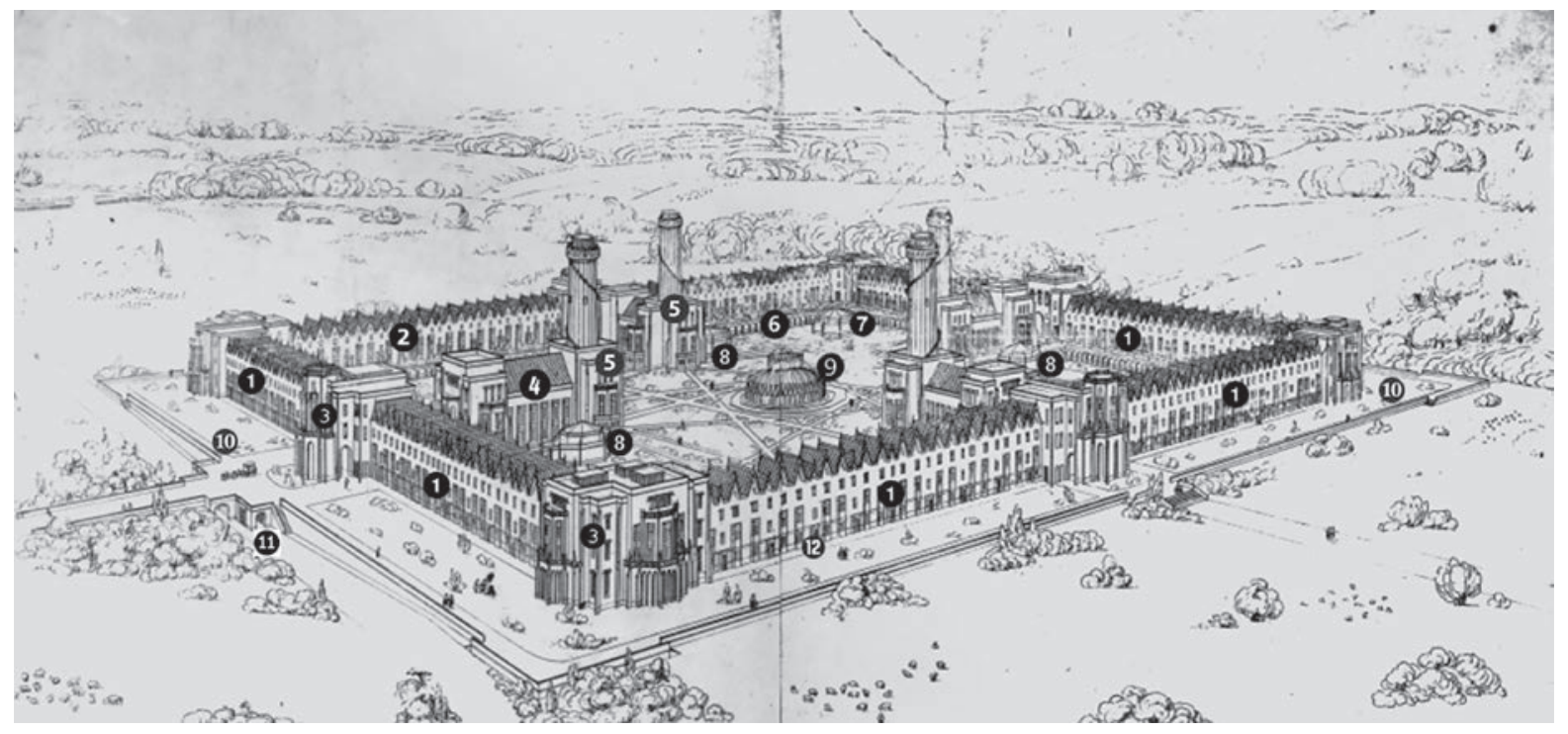

también tendría servicio de agua caliente y fría para cada apartamento, a todas horas. El alumbrado sería con gas y otras innovaciones científicas que reduzcan la mano de obra, se construirían en el sótano general, situado por debajo de todas las plantas de los edificios. El acceso a los sótanos se hará desde los puntos fijos de los edificios centrales y angulares, para evitar la conexión con las viviendas.

\section{Viviendas privadas}

Las viviendas de uso privado estarían situadas en franjas dispuestas entre los edificios centrales y angulares, en cada lado del cuadrilátero, específicamente en el primer y segundo piso. Estarían apareadas y cada una tendría una sala de estar, una recámara y un baño. Además, conectadas coherentemente con las áreas de trabajo mediante circulaciones cubiertas, situadas en un nivel inferior en relación con la entrada general desde la explanada. Según la interpretación de Reps (2002), se asume que dichas circulaciones fueron concebidas como galería de columnas adosadas al edificio en dos niveles, que rodeaban todo el interior del cuadrilátero y que en varias situaciones también funcionaban como puentes de comunicación en la medida que una circulación se convirtiese en la cubierta de la situada en el plano inmediatamente inferior. Estos corredores llevarían a la escalera de cada casa e inclusive a las terrazas que daban hacia el claustro, concediendo así una relación visual y conectiva desde cada casa a cada parte del establecimiento. Esta descripción lleva a evocar proyectos de John Nash ( I 752- I 835) como el de Regent's Park en Londres, el gran parque en cuyos alrededores se establecieron las "terraces", primeras viviendas colectivas de lujo estilo palacete.

\section{Dormitorios}

Con base en la descripción de Raps (2002), el arquitecto Withwell propone una serie de salones para que sean ocupados como dormitorios para solteros y niños; sin conexión con las viviendas o casas privadas debajo de ellos. La ubicación de estos grandes dormitorios es imprecisa. Se sitúan cerca de los grandes puntos fijos que están en los edificios centrales y angulares. Estos espacios fueron pensados para que en determinado momento la totalidad de su área, o parte de esta pudiese convertirse en amplios dormitorios o en su defecto en habitaciones de dimensiones más reducidas. Una vez más aquí los estudiosos ofrecen diferentes versiones que no conducen a una exactitud de la disposición de las áreas de
Figura 4. Dibujo para la Comunidad Owenita en Harmony, Indiana, según interpretación de Robert Owen en 1824

Fuente: Elaboración del autor a partir de Granger Historical Picture Archive y Reps, 2002, Description of an architectural model. http://urbanplanning.library.cornell.edu/DOCS/ whitwell.htm 
vivienda a lo largo de toda la estructura del edifico. Para Benévolo, tres de los lados del perímetro están destinados para viviendas de parejas casadas y el cuarto lado, reservado a dormitorios. Lo cierto es que los niños después de los tres años comerían, dormirían y aprendería en común, norma que ya Owen había establecido en New Lanark.

\section{Volúmenes centrales y esquineros}

Con base en las descripciones escritas y las gráficas elaboradas por Withwell, los edificios centrales se yerguen desde la planta baja hasta la terraza y contienen los grandes accesos al cuadrángulo. Aunque las descripciones disponibles no son muy claras, se asume que tanto en estos como los esquineros se ubican grandes escaleras o puntos fijos que comunican con cada ala de los dormitorios, los apartamentos privados y las salas públicas, contenidos en los demás volúmenes del conjunto. Asimismo, en estos volúmenes también estarían dispuestas las escuelas para infantes, niños y jóvenes, así como las enfermerías. En los primeros pisos, salas de conversación para adultos y las oficinas de administración. Igualmente, estarían las bibliotecas, salas de lectura independientes, imprentas, encuadernadores, museos de historia natural, teatro para conferencias, exposiciones y salas de conciertos.

\section{Edificios públicos centrales dentro del área libre del cuadrilátero, refectorios públicos y otras dependencias}

Después de atravesar cada una de las grandes entradas, se prosigue por la plataforma elevada que a su vez conecta con un nivel de los pórticos y conduce de una u otra forma a los edificios públicos centrales ubicados dentro del paralelogramo. El acceso al hall o vestíbulo se hace por escalinatas con amplios tramos. A cada lado del vestíbulo, se encuentran los comedores para infantes, bebés y niños. Desde el mismo vestíbulo también se llega de frente al gran salón y a un refectorio público, un amplio y elegante espacio, con vanos de piso a techo a cada lado donde se ubican siete ventanales de gran altura que lo iluminan, espacio el cual se propone adoptar cualquier estrategia para reducir el trabajo manual, en particular, lo relacionado con el desplazamiento de las comidas desde las cocinas públicas que se ubican debajo del refectorio para el cual se propone que se realice por medios mecánicos, de una manera rápida, silenciosa y limpia. Además, se propone que los diferentes utensilios usados en el proceso se recojan y se almacenen en elegantes aparadores encajados en nichos. Asimismo, se propone introducir un sofisticado sistema de ventilación tan eficiente como el descrito para los apartamentos. En la parte trasera del refectorio, siempre conectadas al mismo, se ubican zonas especiales de almacenamiento para la cristalería, mantelería, especias y otros condimentos.

\section{Edificios con torres. Relojes iluminados. lluminación desde las torres}

La figura 4 muestra claramente las torres que se elevan desde cuatro volúmenes que son sus bases, en las cuales estarían las fábricas de cerveza, panaderías, lavanderías, lavaderos, etc. Por su parte, las torres estarían destinadas a servir como observatorios y para ese propósito una cómoda escalera de caracol rodeará cada una de ellas, la cual parte desde el techo del edificio trasero que también actúa como base. Aproximadamente a la mitad de cada eje, se colocan relojes iluminados por la noche con gas, visibles a todas horas y desde las áreas internas y los edificios.

\section{Claustro o pórtico y sus terrazas}

Este recorre toda el área interna y forma el límite de los jardines; desde el suelo será una especie de alcoba continua, coronada por una terraza de la misma extensión, ambas amuebladas con asientos que harán de ellas lugares encantadores, ya sea para protegerse del mal tiempo o con fines sociales. Desde la galería y la terraza se plantea una comunicación directa con cada 
zona, particularmente con los baños, gimnasios y jardines. Se observará que, por medio de las galerías que rodean el cuadrángulo interno, se puede mantener la comunicación más perfecta con cada parte del establecimiento cuando hace mal tiempo, sin exponerse a las inclemencias; mientras que la terraza sobre ellas ofrece el mismo acceso y un paseo encantador, bajo circunstancias climáticas más favorables.

\section{Gimnasios}

Sobre las esquinas internas del cuadrilátero se encuentran unos pequeños espacios cubiertos que albergan los gimnasios para bebés, niños y jóvenes de la comunidad, así como para los convalecientes de la enfermería. Están debidamente relacionados con las aulas y a la enfermería. A estos espacios se accede por las circulaciones cubiertas de los claustros y desde los nichos en los ángulos del edificio.

\section{Balnearios - termas}

Cada una de las cuatro divisiones principales de los edificios generales está dotada con baños tibios y fríos, separando los miembros masculinos y femeninos de la comunidad. Estos particulares edificios están a cada lado de los edificios públicos internos centrales, y se accede a ellos a través de los caminos cubiertos de los claustros, y las terrazas que están sobre ellos. Estos baños tendrán un carácter novedoso y peculiar, uniendo la privacidad de la jubilación con el ejercicio que provee en general este tipo de balnearios.

\section{Jardines de recreo e invernadero}

El cuadrilátero debe estar implantado en un terreno con arbustos, jardines floridos, y placenteros campos, científicamente pensados de manera que los mismos contribuyan a la comunicación y sean un medio para inculcar preceptos de orden en todos los niveles. Los paseos deben estar dispuestos de modo que cada uno haga parte de una perspectiva, rematando con algún objeto parte de un edificio, carácter variado y agradable. El centro de los terrenos será ocupado por un invernadero, de unos cien pies de diámetro (30.48 metros), en el cual se albergarán y cultivarán especias exóticas y será climatizado y equipado siguiendo un plan mejorado y novedoso.

\section{La explanada y los alrededores}

La explanada que rodea el edificio general tiene cien pies (30 metros) de ancho, y se extiende sobre el césped y demás terrenos, descritos como sugestivos, con un paseo protegido por una baranda desde donde se divisa el paisaje circundante del establecimiento y la campiña. Se propone una explanada elevada por encima de la superficie base a la cual se llega mediante una amplia rampa de ligera pendiente, de manera que puedan acceder los carruajes que llegan a la edificación. Escalinatas de comunicación se implantarán en todas las esquinas y otros puntos estratégicos de la explanada. Las cuatro fachadas de toda la estructura estarán provistas con árboles frutales, cultivados en espaldera. La extensión de tierra adyacente al edificio y designada a fines agrícolas para el sustento de la comunidad, deberá mantener un efecto pintoresco, en la medida en que los intereses del edificio lo permitan (figura 3).

\section{I.Pasaje subterráneo al sótano y sótano}

Al sótano se accede por una serie de rampas que parten desde la entrada principal de los carruajes y llevan a las cocinas públicas y los almacenes. Esta entrada es suficientemente amplia para la admisión de carruajes para abastecer las diferentes cocinas, tiendas, fábricas de 
cerveza, instalaciones de gas, todos estos elementos concentrados cerca de los cuatro puntos centrales, desde donde se desprenden unas vías férreas que forman un circuito completo de comunicación a lo largo de todo el establecimiento. Sobre estas circulan unos vagones que transportan suministros de combustible, provisiones y otros a los diferentes depósitos, sin que haya interferencia alguna con los jardines, o la explanada o la zona de vivienda e inclusive con las mismas rutas a través de las cuales se evacua todo el material de desecho.

En el sótano de los edificios centrales interiores, debajo del gran hall y los refectorios, se encuentran las cocinas públicas, fregaderos, despensas, oficinas para la distribución de víveres, etc. Alrededor de las bases de las torres están dispuestas las fábricas de cerveza, las gasolineras, los lavaderos, las lavanderías, las maquinarias de vapor para el suministro en la cocina y otros aparatos para evacuar todo el humo y el vapor que se acumula en estos puntos y se lleva hacia arriba a través de los grandes conductos de las torres; los cuales permiten el cómodo acceso de los trabajadores a cualquier parte y en todo momento, en caso de que sean necesarios trabajos de reparación o limpieza. Las bóvedas para la estiba de licores de malta, combustible y otras mercancías pesadas, son de grandes y espaciosas dimensiones y están situadas en proximidad de aquellos lugares donde su servicio se requiere de manera más inmediata.

\section{Andén perimetral}

Se trata de una circulación peatonal a lo largo de todo el perímetro del edificio que permitía la conexión visual de los espacios del primer piso con el exterior.

\section{EL OCASO DE LA UTOPÍA DE OWEN}

Las páginas anteriores han descrito en modo sucinto la majestuosidad de la idea de Owen. Sin duda, un modelo de ciudades futuristas para esa época. Lamentablemente, la falta de soberanía individual y de propiedad privada llevó a que ocurriera lo vaticinado por muchos observadores: la comunidad estaba condenada al fracaso. En 1826 se reafirman los grupos disidentes, y por ende la separación y la reorganización de New Harmony, la cual se disuelve definitivamente en 1829. Los bienes fueron devueltos a su uso privado y Owen culpó al hombre de ser tan "egoísta" de no poder vivir sin propiedad privada. Gordon (1993) relata que a su regreso a Estados Unidos, en 1828, Owen admitió que el experimento había fracasado:

He ensayado aquí un nuevo rumbo, en la esperanza de que cincuenta años de libertad política hubiesen preparado a la población americana a gobernarse a sí misma. Proporcioné la tierra, las casas y gran parte del capital... pero la experiencia ha demostrado que es prematuro tratar de unir a extraños que no han recibido previamente una educación con tal objetivo, de manera que puedan practicar múltiples actividades en su interés común y vivir juntos como si formaran parte de una misma familia (Discurso a los habitantes de New Harmony 1828).

\section{El legado de Owen}

Sin lugar a duda, Owen consolidó una nueva visión de la sociedad gracias a sus propias ideas sobre la organización social, el carácter del ser humano y la educación. Dejó muy en claro que una nueva forma de vida puede darse a partir de un régimen más humano que dignifique a los trabajadores. Su experimento social esperaba cristalizar un nuevo orden 
con trabajadores felices, formados, unidos y prósperos; a través de un aprendizaje racional. En otras palabras, un gobierno de la industria con beneficios a los empleados.

Muy ambicioso citar todos los logros de Owen, pero sin duda desde su experiencia New Lanark se sentaron las bases para un nuevo sistema de organización laboral. Es así que prohíbe el trabajo infantil, reduce la jornada laboral, otorga licencias de trabajo por enfermedad (incapacidades) e impone la educación para todas las clases. De dominio público es el hecho de que Owen crea el primer centro preescolar en el Reino Unido. Además, excluye la religión de sus instituciones, crea bibliotecas para los empleados e instituciones culturales para los obreros adultos. Esto lo denotan sus premisas como "el Estado con el mejor sistema nacional de educación será el mejor gobernado”, "el fin de la educación es que el hombre sea bueno, sabio y feliz" y "la sociedad es el producto de la formación de sus miembros”. Con New Harmony vislumbraba un escenario ideal que contribuyera al carácter de los hombres, resultado concordante de un adecuado medio intelectual y moral para vivir y trabajar. A esto se le suma sus ideales de trabajo comunitario, en pos de una autosostenibilidad comunitaria. Es cierto que esta utopía no funcionó, pero también es claro que sentó las bases del cooperativismo moderno y que numerosas cooperativas británicas de consumo surgieron influidas por sus ideas.

De la obra de Owen surgen también muchas analogías hechas por estudios e investigadores del urbanismo, los cuales identifican en las propuestas de Ildefonso Cerdá (I815-1876), Ebenezer Howard (1850-1928), Frank Lloyd Wright (1867-1959) o el mismo Le Corbusier (1887-1965), muchos elementos concomitantes con aquellas hechas por los preurbanistas, entre estos Robert Owen (I77I-1858) y Charles Fourier (I722-I837). Difícil establecer en qué medida lo estudiaron o lo siguieron, pero lo que es indiscutible es que en los diseños de los urbanistas del siglo XX está también presente una respuesta a la necesidad de solucionar los problemas sociales, ligados a la calidad de vida de las ciudades y demás procesos de la urbanización. Un análisis comparativo demuestra a priori grandes similitudes entre preurbanistas y urbanistas, más en el componente técnico que en lo social, pues la humanidad siempre ha evolucionado y lo ha seguido haciendo desde la Revolución Industrial hasta nuestros días. En cualquier caso, Owen entra sin duda en el grupo de los "profetas del progreso" gracias a su socialismo utópico y a sus detallados planes para construir comunidades utópicas, planes para una revolución desde el punto de vista social y también arquitectónico que anticiparon, como ya se mencionó, el trabajo de muchos urbanistas.

Aunque no se llegó a construir ninguno de sus paralelepípedos queda también un buen número de elementos tangibles considerados patrimonio de Owen, entre estos su producción literaria con una serie de teorías sobre la naturaleza humana, que puede ser interpretadas como ideas fundadoras del socialismo. Obras como: A new view of society or Essays (1813-1814) y el The book of the new moral world (1836-1844) son prueba de esta visión. Quedan además dos testimonios urbanos y arquitectónicos de su intención, cada uno con rasgos particulares inherentes a grupos sociales específicos. El primero, New Lanark donde llevó a cabo sus primeros experimentos sociales. El segundo, la New Harmony de hoy, una pequeña ciudad que rememora a Owen y su legado.

En este orden de ideas, es más que plausible darle a Owen el mérito por haber sentado las bases de nuevas teorías sociales. La repercusión de sus ideas en la historia es aún vigente y aun recordada por los historiadores y analistas que escudriñan sobre la evolución del concepto de socialismo en Gran Bretaña. De hecho, a Marx y Engels se les señala como sus sucesores, pues crearían la teoría de un sistema social que hoy está implantado en muchos países.

M 77 REVISTA M VOL. 14 ENERO - DICIEMBRE 2017 • FACULTAD DE ARQUITECTURA • UNIVERSIDAD SANTO TOMÁS COLOMBIA 


\section{CONCLUSIONES}

Robert Owen hace parte de los idealistas del preurbanismo, personas que concebían entornos imaginarios en una posible realidad, circundada de novedades en arquitectura, ciudad, arte, literatura, filosofía y política. Interesante posición que lleva a pensar el valor de la utopía que invita a leer a Mumford (2010).

Este artículo es un homenaje a Owen y a todos aquellos utopistas que pusieron sobre la mesa sus modelos de ciudades ideales o a las múltiples propuestas, dominio virtual de sus ideas y que son en su esencia intentos por recomponer la ciudad con criterios de orden, unidad y prefiguración alteradas en pro de la Revolución Industrial, importante fenómeno que ha servido como inspiración para las investigaciones de las cuales se desprende este trabajo.

En la introducción del artículo se esbozan nombres, posiciones y postulados que han dado origen a corrientes teóricas que orientan el análisis sobre los procesos de configuración urbana y sus efectos sociales y que bien pueden servir de base a ulteriores investigaciones. El orden de análisis histórico establecido por Françoise Choay puede ser también el fulcro de futuros estudios sobre las teorías urbanas, ya que cada teoría asume diversos sentidos según la visión que se tenga de la ciudad y su componente social. Para Choay hay claras diferencias en términos de unidad, orden y diseño del proyecto y son estos parámetros los que dan origen a tres grandes corrientes o posiciones: Progreso y Técnica; Historia y Cultura; y Naturaleza. Evidentemente, todas involucradas en mayor o menor idea en la propuesta de Owen, cristalizada en el proyecto del arquitecto Withwell: un lugar cerrado en su perímetro, pero con accesos por cada uno de sus lados, con torres de sus esquinas para ser vigilado; con alturas diferentes en las edificaciones situadas en su interior para denotar la capacidad o el poder productivo de una comunidad; con cuatro chimeneas que no emanarían desde su punto más alto vapores contaminantes como las chimeneas de las fábricas de la Revolución Industrial, sino por el contrario, "estelas de progreso", muy diferentes a las que se elevaban por encima de los campanarios de las ciudad medievales europeas.

A pesar de que la New Harmony de Owen duró alrededor de dos años (I825-1826) y que se disolvió por problemas internos agravado por la presión externa, su promotor confiaba en que la solución vendría de la propia sociedad. Loable su intención de valerse de una propuesta arquitectónica con sus relativas implicaciones territoriales para conseguir una sociedad mejor, un modelo que hoy en día solo se muestra como parte de la ficción y que demostró que el sindicato de masas en ámbito estatal es posible y que se pueden plantear alternativas al sistema capitalista como cooperativas de producción. Es por esto que Owen se dedicó a escribir y defender su visión de cooperativismo antes de su muerte y a atacar las tradicionales posiciones de muchas instituciones e inclusive de la religión y de la herencia, todas, según él, limitantes de la libertad del ser humano. Hoy en día, la ciudad New Harmony sigue rindiendo culto a Robert Owen, busca ser reconocida como el centro que despertó gran interés social y educativo gracias al aporte de este reconocido preurbanista.

\section{REFERENCIAS}

BBC. (2017). How utopian shaped the world. Recuperado de http://www.bbc.com/ culture/story/20160920-how-utopia-shaped-the-world.

Benévolo, L. (1994). Los orígenes del urbanismo moderno. Madrid: Celeste. 
Coates, C. (200I). Utopía Británica. London: Diggers \& Dreamers Publications.

Choay, F. (197I). El urbanismo: Utopías y realidades. Chicago: Turabian.

Donnachie, I. (2017). Utopian Designs: The Owenite Communities", Spaces of Utopia: An Electronic Journal, No. 6, 19-34. Autumn/Winter. Recuperado de http://ler.letras.up.pt/ uploads/ficheiros/434I.pdf.

Eighteenth Century Birmingham. (20I2). Thomas Stedman Whitwell. Recuperado de http://mappingbirmingham.blogspot.com/2012/06/thomas-stredman-whitwell.html.

Engels, F. (2009). Del socialismo utópico al socialismo científico. Córdoba: El Cid Editor.

Gordon, P. (1993). Perspectivas: Revista Trimestral de Educación Comparada, XXIV (I-2), 279-297. París: UNESCO: Oficina Internacional de Educación.

Granger Historical Picture Archive. 2017. Owenite Community, 1824. Design for an Owenite community by Stedman Whitwell, the architect who accompanied Robert Owen to New Harmony, Indiana in 1824. Recuperado de https://www.granger.com/

Mumford, L. (2010). Historia de las utopías. Madrid: Ed. Pepitas de Calabaza.

New Lanark World Heritage Site. (2018). Portrait of Robert Owen, social reformer and owner of New Lanark Mills from 1800 to 1825. Recuperado de https://artuk.org/discover/ artworks/robert-owen-177| |858-189632.

Owen, R. (1975). Self-Supporting Home Colonies: A development of the principles and plans on which to establish self-supporting home colonies. Nueva York: AMS Press.

Owen, R. (I8I7). Report to the Committee of the Association for the Relief of the Manufacturing and Labouring Poor, referred to the Committee of the House of Commons on the Poor Laws. Londres: Watts R.

Reps, J. (2002). Description of an architectural model. New York: Department of City and Regional Planning, West Sibley Hall, Cornell University. Recuperado de http://urbanplanning. library.cornell.edu/DOCS/whitwell.htm

Reps J. (2002). Urban Planning, I794-1918: An International Anthology of Articles, Conference Papers, and Reports. Cornell Univeritsy. Recuperado de http://urbanplanning.library. cornell.edu/DOCS/owen_I7.htm.

Visit Heritag. The Home of Heritage. (2017). New Lanark Mills World Heritage Site Recuperao de https://www.visitheritage.co.uk/whats-on/new-lanark-mills-world-heritagesite-p20978II.

Whitwell, S. (1830). Description of an architectural model: from a design by Stedman Whitwell, Esq. for a community upon a principle of united interests, as advocated by Robert Owen, Esq. London: Hurst Chance \& Co. and E. Wilson. 